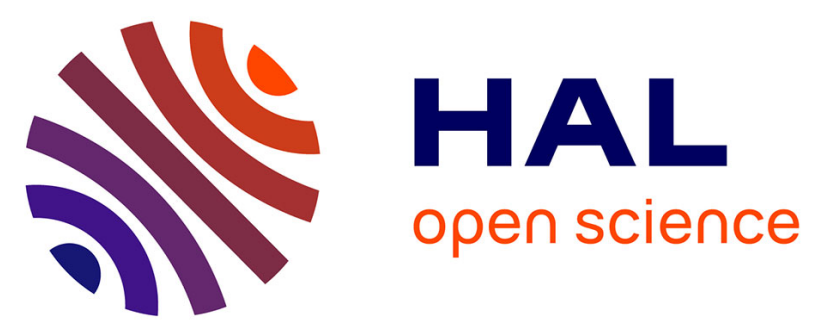

\title{
Influence of the contact angle of silica nanoparticles at the air-water interface on the mechanical properties of the layers composed of these particles
}

Dominique Langevin, Bingbo Wei, Duyang Zang, Bernard Binks, Emmanuelle Rio, Giles Delon

\section{To cite this version:}

Dominique Langevin, Bingbo Wei, Duyang Zang, Bernard Binks, Emmanuelle Rio, et al.. Influence of the contact angle of silica nanoparticles at the air-water interface on the mechanical properties of the layers composed of these particles. Molecular Physics, 2011, pp.1. 10.1080/00268976.2010.542778 . hal-00686160

\section{HAL Id: hal-00686160 https://hal.science/hal-00686160}

Submitted on 8 Apr 2012

HAL is a multi-disciplinary open access archive for the deposit and dissemination of scientific research documents, whether they are published or not. The documents may come from teaching and research institutions in France or abroad, or from public or private research centers.
L'archive ouverte pluridisciplinaire HAL, est destinée au dépôt et à la diffusion de documents scientifiques de niveau recherche, publiés ou non, émanant des établissements d'enseignement et de recherche français ou étrangers, des laboratoires publics ou privés. 


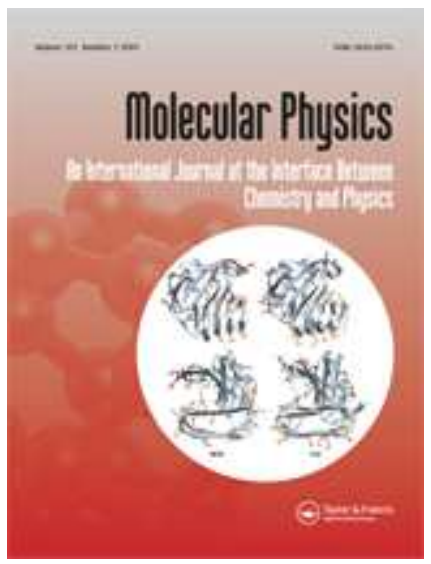

\section{Influence of the contact angle of silica nanoparticles at the air-water interface on the mechanical properties of the layers composed of these particles}

\begin{tabular}{|c|c|}
\hline Journal: & Molecular Physics \\
\hline Manuscript ID: & TMPH-2010-0379.R1 \\
\hline Manuscript Type: & Special Issue paper - In honour of Bob Evans \\
\hline $\begin{array}{r}\text { Date Submitted by the } \\
\text { Author: }\end{array}$ & 05-Nov-2010 \\
\hline Complete List of Authors: & $\begin{array}{l}\text { Langevin, Dominique; Université Paris Sud 11, Laboratoire de } \\
\text { Physique des Solides } \\
\text { Wei, Bingbo; Northwestern Polytechnical University } \\
\text { Zang, Duyang; Northwestern Polytechnical University } \\
\text { Binks, Bernard; University of Hull } \\
\text { Rio, Emmanuelle; université paris sud } \\
\text { delon, Giles; Université de Liege }\end{array}$ \\
\hline Keywords: & nanoparticles monolayers, contact angle, surface rheology, foams \\
\hline
\end{tabular}

\section{SCHOLARONE ${ }^{\text {M }}$ \\ Manuscripts}




\title{
Influence of the contact angle of silica nanoparticles at the air-water interface on the mechanical properties of the layers composed of these
} particles

\author{
D.Y. Zang, ${ }^{1,2}$ E. Rio, ${ }^{1}$ G. Delon, ${ }^{1,4}$ D. Langevin, ${ }^{1}$ B. $\mathrm{Wei}^{2}$ and B.P. Binks ${ }^{3}$ \\ 1. Laboratoire de Physique des Solides, Université Paris-Sud 11, UMR CNRS 8502, Bâtiment \\ 510, 91405 Orsay cedex, France \\ 2. Department of Applied Physics, Northwestern Polytechnical University, Xi’an, 710072, \\ China \\ 3. Surfactant \& Colloid Group, Department of Chemistry, University of Hull, \\ Hull, HU6 7RX, UK \\ 4. Permanent address : GRASP, Institut de Physique, Bâtiment B5, Université de Liège, \\ B-400 Liège, Belgium
}

\begin{abstract}
:
We have studied the properties (surface pressure, compression and shear moduli, texture) of silica nanoparticle layers at the air-water interface. Particle hydrophobicity or equivalently the contact angle between particles, air and water, is the main factor that influences surface organisation and surface elastic moduli. The surface layers are denser for particles of higher hydrophobicity. The compression and shear moduli, as well as the yield and melt strains, present a maximum for contact angles around $90^{\circ}$. The dependence of mechanical properties on particle hydrophobicity is closely related to the foamability and stability of the foams made from dispersions.
\end{abstract}




\section{Introduction}

It is now well known that aqueous foams can be stabilized solely by partially hydrophobic particles of nano- or micrometer size [1-5]. They are the analogue of Pickering emulsions [6, 7], which were discovered at the beginning of the $20^{\text {th }}$ century. In order to stabilize foams or emulsions, the particles should adsorb at the air-water or oil-water interfaces, which implies that the contact angle $\theta$ of the particles with the interface, measured into water, should be neither zero (very hydrophilic particles) nor $180^{\circ}$ (very hydrophobic particles), see Figure 1(a): indeed, the adsorption energy $\mathrm{W}$ is :

$$
\mathrm{W}=\pi \gamma R^{2}(1 \pm \cos \theta)^{2}
$$

assuming spherical particles of radius $R, \gamma$ being the air-water or oil-water interfacial tension. The positive sign in brackets corresponds to $\theta<90^{\circ}$, the negative sign to $\theta>90^{\circ}$. As soon as $\theta$ is slightly above 0 or below $180^{\circ}, \mathrm{W}$ is large, well above the thermal energy $\mathrm{k}_{\mathrm{B}} \mathrm{T}$, and adsorption is not only favoured but irreversible. W is maximum for $\theta=90^{\circ}$ and of order $10^{3} k_{\mathrm{B}} T$ for particles with radius of about $10 \mathrm{~nm}$ and $\gamma \sim 40 \mathrm{mN} / \mathrm{m}$. It is not easy to measure contact angles, especially for particles of nanometre size. Commonly used techniques include: compression of the particles into a flat plate and observations of deposited water drops [8], colloidal probe AFM [9], film trapping [10], gel trapping [11] and a film calliper method [12]. Figure 1(b) shows measurements of the contact angle of a water drop in air on plates obtained by compaction of fumed silica particles made increasingly hydrophobic by a silanisation treatment [8].

(a)

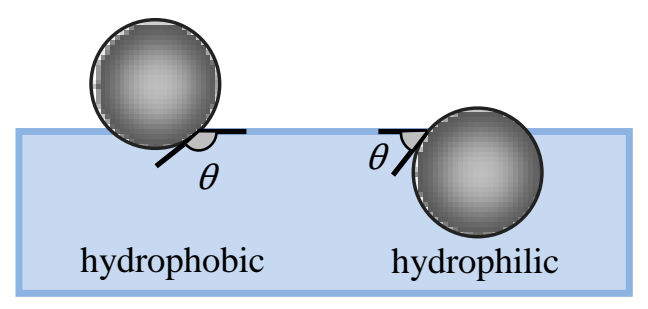

(b)

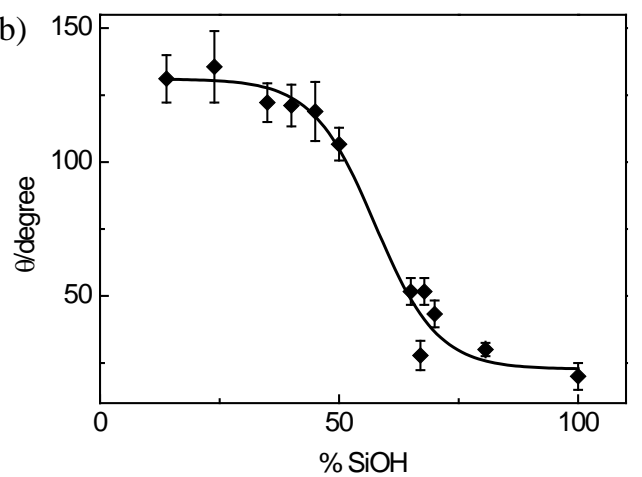

Figure 1. (a) Schematic representation of a particle at the air-water surface and (b) measurements of the contact angle of water in air for surfaces of fumed silica particles versus the percentage of silanol groups $\mathrm{SiOH}$ (adapted from ref. 8). 


\section{Materials and methods}

\subsection{Materials}

The silica particles were kindly provided by Wacker-Chemie (Germany) and were used as received. The surfaces of the particles were modified by reaction of the surface silanol groups with dichlorodimethylsilane. In this study, we used particles with a relative SiOH content x of 20, 34, 51, 62, 78 and 100\%, in which the hydrophobicity increases with decreasing $\mathrm{SiOH}$ content. The primary particles are nearly spherical and of approximately $20 \mathrm{~nm}$ in diameter. The dispersions were prepared by adding amorphous fumed silica powder into isopropyl alcohol to a final concentration of $1 \mathrm{~g} / \mathrm{L}$ and applying sonication for $30 \mathrm{~min}$. We used an 
ultrasonic probe (Ultrasonic Processor) operating at $20 \mathrm{kHz}$ with an amplitude of $45 \%$ of the maximum in order to have a stable dispersion and to avoid the formation of particle aggregates. The hydrodynamic radius $R_{h}$ of the particles in the dispersion has been measured by dynamic light scattering [13]. Upon increasing $\mathrm{x}, R_{h}$ decreases from $87 \mathrm{~nm}$ to $72 \mathrm{~nm}$. The particles were found fairly monodisperse, the polydispersity index being between 0.11 and 0.21. The dispersions were re-sonicated for $10 \mathrm{~min}$. just before each experiment.

Water used in the experiments was from a Millipore-Q instrument (resistivity $=18 \mathrm{M} \Omega \mathrm{cm}$ ). All the experiments were performed at room temperature $\left(\sim 22{ }^{\circ} \mathrm{C}\right)$. The particle dispersion was spread onto the air-water interface drop by drop from a microlitre syringe.

\subsection{Langmuir trough}

The surface pressure-surface concentration $(\Pi-\Gamma)$ isotherms were recorded on a Nima 601BAM trough with total area $A=500 \mathrm{~cm}^{2}$. The barrier speed, $\mathrm{d} A / \mathrm{d} t$, can be accurately controlled in the experiments. Two Wilhelmy plates oriented parallel and perpendicular to the barrier orientation measure the surface pressure in both directions[16]. They were positioned in the middle of the trough at a distance ca. $2 \mathrm{~cm}$ from the borders. The Langmuir trough was cleaned with ethanol and pure water before each experiment and by acetic acid if the type of particles was changed. Before spreading the particles, the surface of pure water was compressed and it was checked that the final surface pressure did not exceed $0.3 \mathrm{mN} / \mathrm{m}$, ensuring the absence of contamination. After spreading a certain volume of particle dispersion onto the water surface with a micro-syringe, we waited for $30 \mathrm{~min}$. to allow the solvent to evaporate. During each experiment, the two barriers moved simultaneously and the speed was kept constant. Two compression speeds were used, 7 and $25 \mathrm{~cm}^{2} / \mathrm{min}$.

\subsection{Successive deposition method}

Measurements were carried out in the Langmuir trough without compression (keeping the barriers fixed, the effective area being $180 \mathrm{~cm}^{2}$ ) and in a homemade circular teflon trough, in order to study the monolayer properties under extremely small compression rates, i.e. to approach the equilibrium state. The surface tension in the circular trough was monitored by a single Wilhelmy plate, positioned at the center of the trough. In all these experiments, the area 
of the monolayer was constant. The surface concentration of particles $\Gamma$ was varied by successive addition of small volumes of particle dispersion. Before spreading the dispersion on the surface, the surface tension of pure water was measured: a value of $72.5 \mathrm{mN} / \mathrm{m}$ ensures the purity of the water and the cleanliness of the trough. When the particle dispersion was spread onto the surface, the surface pressure first increased, then slowly decayed, due to relaxation processes in the monolayer. The values of the surface pressure obtained $100 \mathrm{~min}$. after spreading the dispersion did not change further (within experimental accuracy, $\sim 0.1$ $\mathrm{mN} / \mathrm{m}$ ) during a few hours and were assumed to correspond to equilibrium. Note however that a decrease of the order of $1-2 \mathrm{mN} / \mathrm{m}$ was still seen over much longer time scales ( $\sim$ day).

\subsection{Interfacial shear rheometer}

The shear measurements were performed with a rheometer (Anton Paar Physica MCR 301) with an embedded interfacial rheology system (IRS). The interfacial rheology cell is based on a bicone geometry [17]. The interfacial shear moduli and viscosity were determined after subtraction of the contribution of the bulk water using the instrument software. In each experiment, the particle dispersion was spread onto the air-water interface in the cell. The bicone was positioned after $30 \mathrm{~min}$. in order to allow for evaporation of the alcohol.

\subsection{Brewster angle microscopy}

During the compression-expansion cycles, the particle monolayer texture was observed with a Brewster Angle Microscope (MiniBAM, NFT-Nanofilm Technology, Göttingen). The BAM is equipped with a high-power visible laser diode (wavelength $688 \mathrm{~nm}$ ), a polarizer and an analyzer. The images taken at the Brewster angle of $53.1^{\circ}$ were recorded by means of a video recorder. In the images, white parts signify the domains covered by particles while dark parts are the bare water surface.

\section{Results and Discussion}

\subsection{Textural evolution}

During continuous compression, the particle layers evolve from isolated islands (inhomogeneous layer) to homogeneous layers and finally to bucked layers [16]. These 
textural observations are similar to what we reported earlier for the same particles at the air-water interface by BAM [18]. Fractures are generated during expansion and are slowly healed by compression, also in line with earlier studies $[18,19]$.

(a) $20 \% \mathrm{SiOH}$
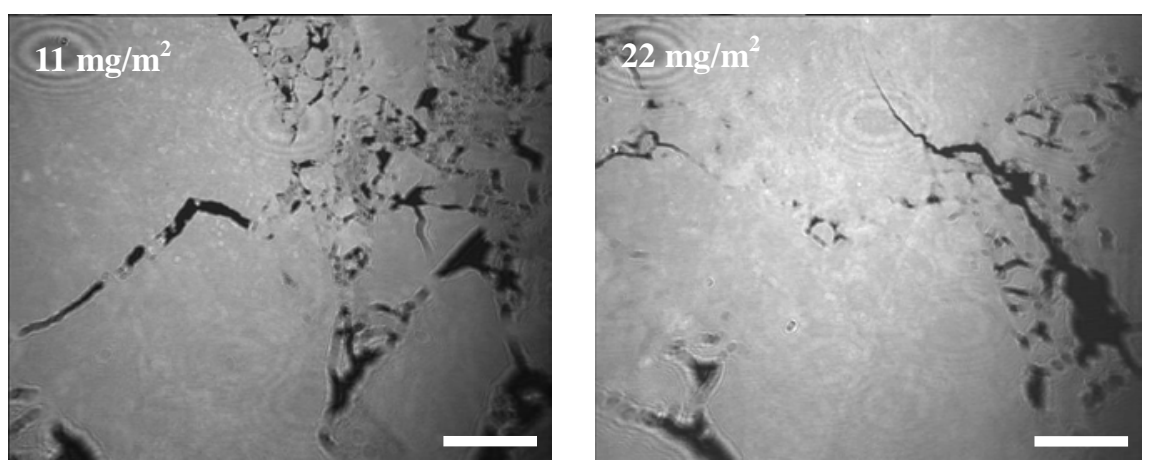

(b) $34 \% \mathrm{SiOH}$
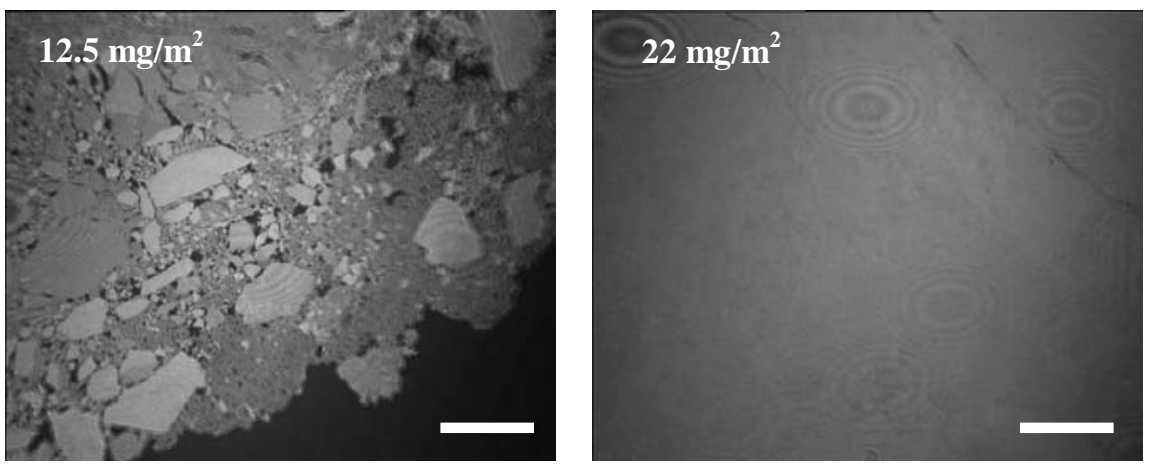

(c) $51 \% \mathrm{SiOH}$
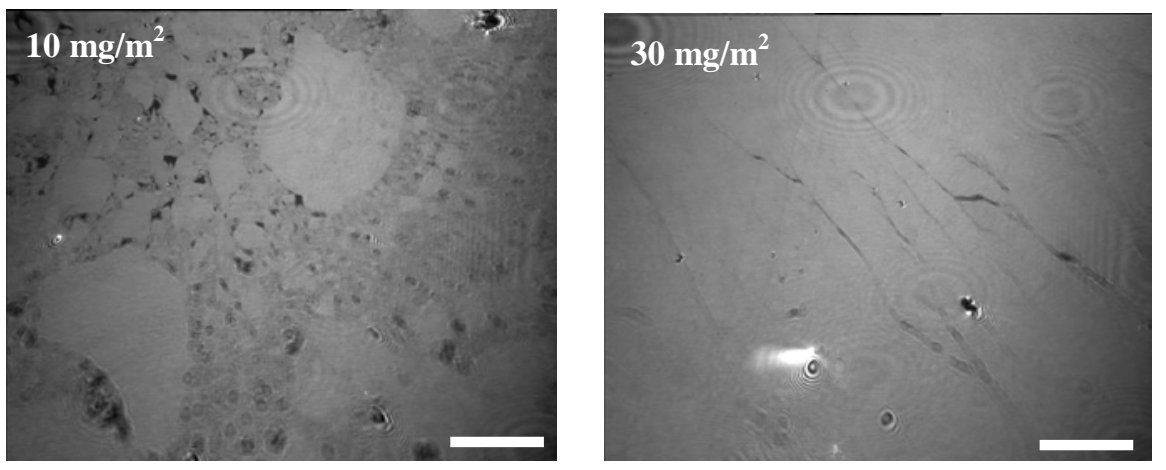

(d) $78 \% \mathrm{SiOH}$
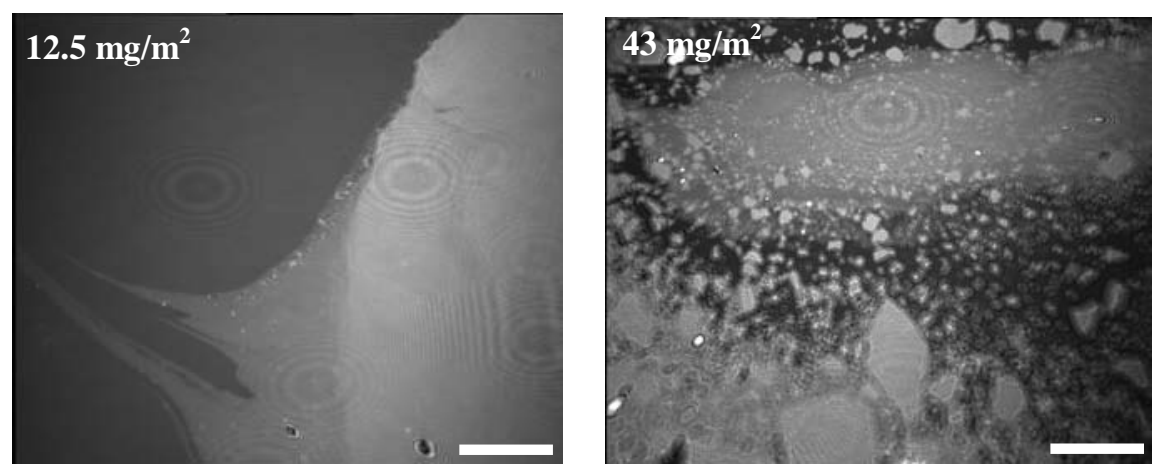

Figure 2. Textural evolution of successively deposited silica particle layers with increasing surface concentration (left to right) and decreasing hydrophobicity (from (a) to (d)). The scale bars correspond to $1 \mathrm{~mm}$. 


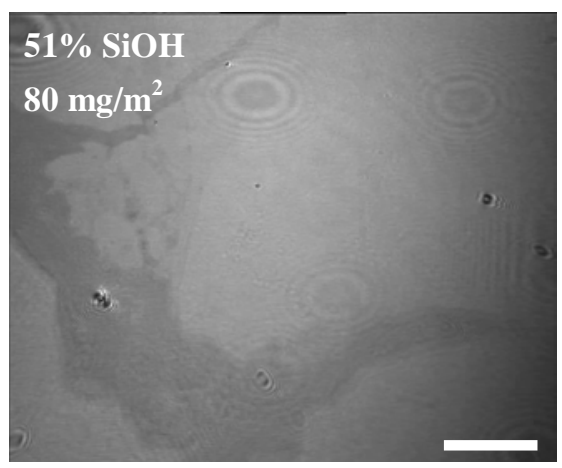

Figure 3. Image of a deposited silica particle layer ( $51 \% \mathrm{SiOH})$ for $\Gamma=80 \mathrm{mg} / \mathrm{m}^{2}$. Scale bar $=1 \mathrm{~mm}$.

\subsection{Surface pressure isotherms}

\subsubsection{Anisotropic effect}

The variation of the surface pressure with surface coverage was monitored by two orthogonal Wilhelmy plates during three compression-expansion cycles. Figure 4(a) illustrates the variation of $\Pi_{/ /}$with surface concentration $\Gamma$, called the "pressure isotherm”. The difference $\Delta \Pi=\Pi_{\| /}-\Pi_{\perp}$ is shown in Figure 4 (b). $\Delta \Pi$ is non-zero at large $\Gamma$, revealing the existence of a finite shear modulus and the fact that the monolayers are out of equilibrium [16,20-22].

In order to check for the reproducibility of the isotherms, each experiment was repeated 
several times, keeping the same spreading conditions. The different curves never coincide exactly, but they are shifted along the horizontal compression axis by at most $5 \mathrm{mg} / \mathrm{m}^{2}$ and the maximum pressures vary by at most $\pm 2-3 \mathrm{mN} / \mathrm{m}$.
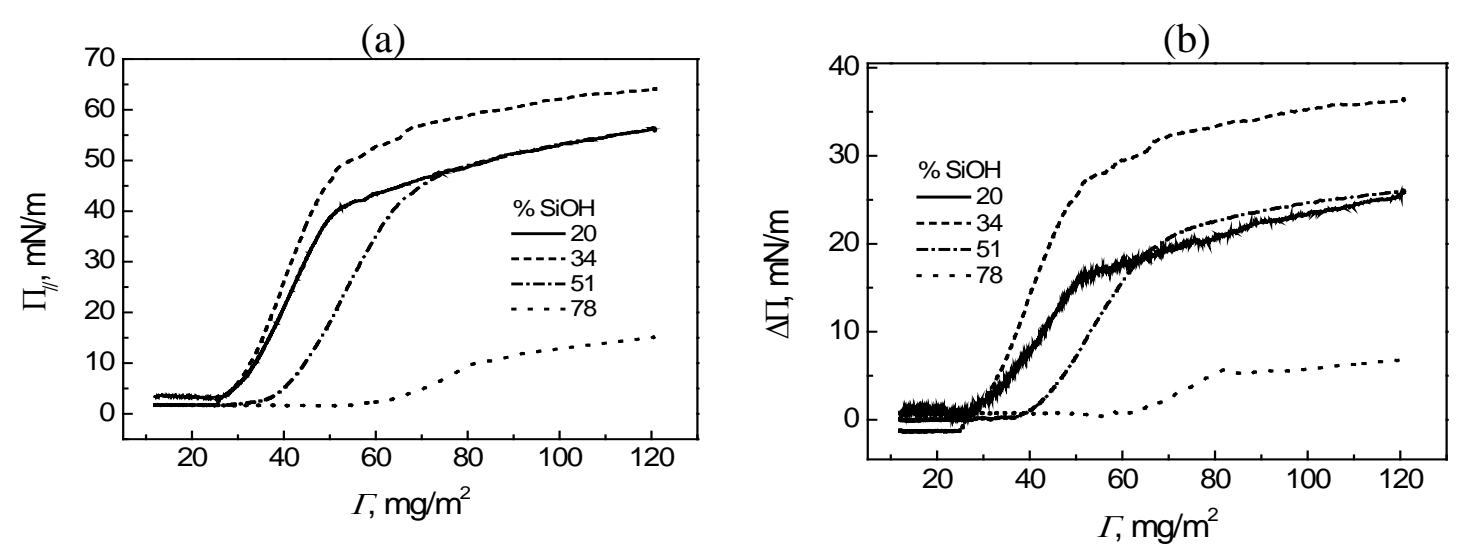

Figure 4. Surface pressure anisotropy for silica particle layers of different hydrophobicity (given) measured by two Wilhelmy plates, respectively parallel $\left(\Pi_{\|}\right)$and perpendicular to the barriers $\left(\Pi_{\perp}\right)$. The barrier speed is $25 \mathrm{~cm}^{2} / \mathrm{min}$ and the fully open area $500 \mathrm{~cm}^{2}$. (a) $\Pi_{/ /} v s \Gamma$. (b) Surface pressure difference, $\Delta \Pi=\Pi_{\| /}-\Pi_{\perp}, v s \Gamma$.

The isotherms strongly depend on the hydrophobicity of the particles. The closer $\mathrm{x}$ is to $34 \%$, the higher the surface pressure and the difference $\Delta \Pi$. The optimum contact angle of $90^{\circ}$ leading to the highest adsorption energy corresponds rather to $\mathrm{x} \sim 50 \%$ according to Figure 1(b). However, it is possible that the method of measurement used in ref. 8 introduces artifacts and shifts along the $\mathrm{x}$-axis of the curve. The accuracy of the direct ellipsometry measurements is unfortunately not sufficient to confirm the shift.

\subsubsection{History dependence}

Surface pressure isotherms were measured at two different barrier speeds: 7 and $25 \mathrm{~cm}^{2} / \mathrm{min}$. and are compared in Figure 5: $\Pi$ decreases slightly with decreasing barrier speed. The difference confirms that continuous compression produces monolayers out of equilibrium. It also reveals that a long period of time is needed to reach equilibrium. The behavior observed is similar for the different particles.

In order to approach better the equilibrium state of the particle monolayer, successive particle deposition experiments were carried out. The surface pressures of the deposited layer are 

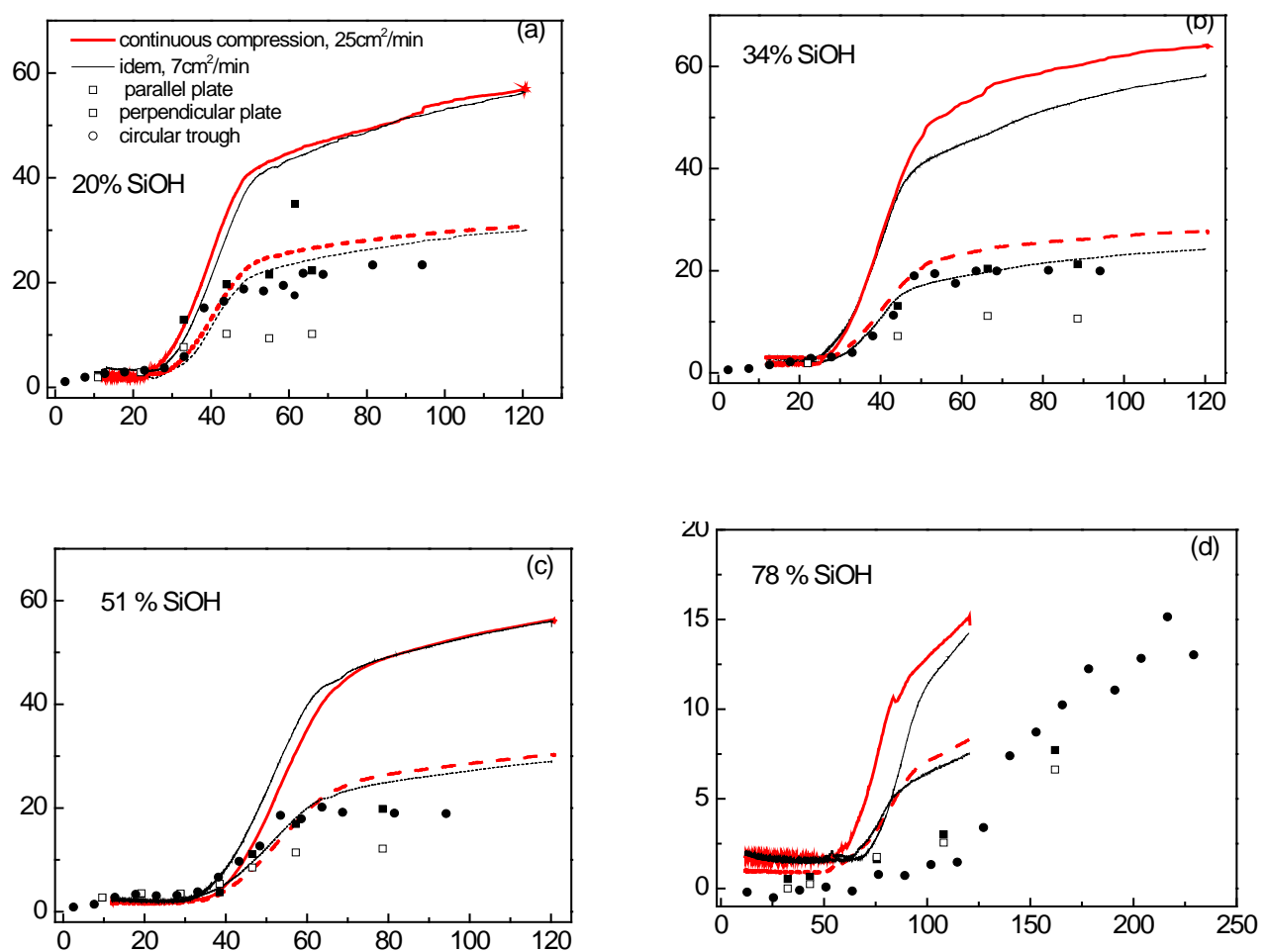

Figure 5. History dependence during the first compression of particle monolayers with different hydrophobicity._The solid lines correspond to data obtained from the plate parallel to the barriers, the dashed lines, to the plate perpendicular. The red lines are for a compression speed of $25 \mathrm{~cm}^{2} / \mathrm{min}$, the black curves to $7 \mathrm{~cm}^{2} / \mathrm{min}$. The points correspond to data from successive spreading experiments: in the Langmuir trough $\Pi_{/ /}\left(\right.$filled square $\mathbf{~}$ ) and $\Pi_{\perp}$ (open square $\square$ ); in the circular trough (filled circle •). (a) $20 \% \mathrm{SiOH}$, (b) $34 \% \mathrm{SiOH}$, (c) $51 \% \mathrm{SiOH}$, (d) $78 \% \mathrm{SiOH}$.

These latter measurements can be regarded as compression of the monolayer at extremely low speed. The surface pressures should therefore be closer to equilibrium values. When $\Gamma$ is small, $\Pi_{/ /}$and $\Pi_{\perp}$ in the Langmuir trough and $\Pi$ in the circular trough are very close to each other. This is because the monolayer is not dense and the relaxation time is short. At large $\Gamma$, the differences between $\Pi_{\| /}$and $\Pi_{\perp}$ become large revealing that the existence of a shear modulus. The differences between compressed and deposited layers suggest that the deposited layers are less compact and that compression has a consolidating effect, as in 3D granular media [23]. Deposited layers also show unexpected anisotropic effects (squares, Figure 5) : spreading possibly leads to an inhomogeneous layer and internal stresses are still present.

\subsubsection{Hysteresis}


In the first compression-expansion cycle, hysteresis has been found depending on whether the particles are hydrophobic or hydrophilic. This behavior is influenced by the particle hydrophobicity. Figure 6 illustrates two different types of hysteresis behavior.

(a)

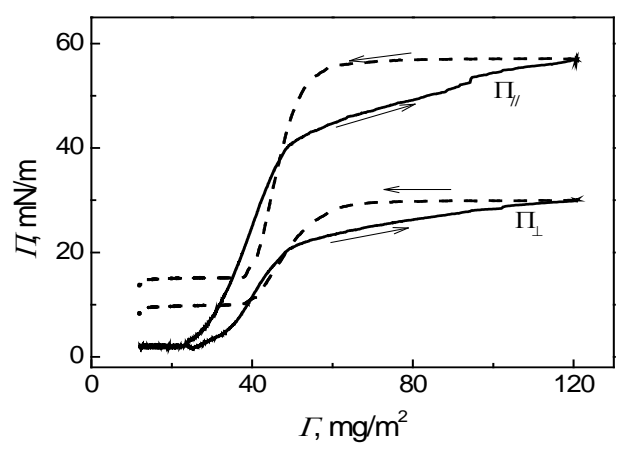

(b)

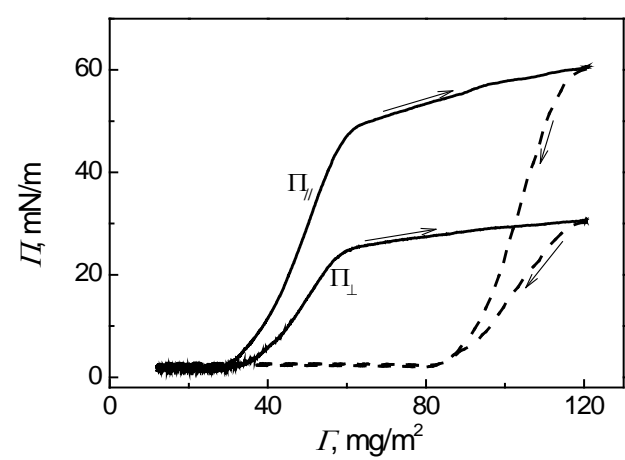

Figure 6. Hysteresis between compression and expansion during the first cycle for monolayers of silica particles possessing (a) $20 \%$ and (b) $51 \% \mathrm{SiOH}$ on their surfaces. The barrier speed is $25 \mathrm{~cm}^{2} / \mathrm{min}$. The arrows indicate the direction of change of $\Gamma$.

Note that similar results were reported before in ref. 18 but using a single Wilhelmy plate. For particles with $20 \% \mathrm{SiOH}$ (the most hydrophobic), during expansion, i.e. decreasing $\Gamma$, the surface pressure remains at a constant high level until $\Gamma=60 \mathrm{mg} / \mathrm{m}^{2}$ and then decreases sharply when $\Gamma$ is smaller. Surprisingly, the surface pressure never comes back to zero (Figure 6(a)) even if the area is back to its initial value. Both Wilhelmy plates detect a quite high residual pressure, $c a .10 \mathrm{mN} / \mathrm{m}$ with the perpendicular plate and $15 \mathrm{mN} / \mathrm{m}$ with the parallel one. This means that the mechanical energy stored in the monolayer during compression cannot be totally released. Indeed, the islands seen by BAM remain weakly interconnected and the layer never comes back to its initial state.

The hysteresis behavior of the monolayers with 34, 51, 78 and 100\% SiOH are similar to each other and quite different to that for $20 \% \mathrm{SiOH}$ (Figure 6(b)). During the first expansion the surface pressure decreases quickly and falls to zero. No plateau occurs in this case. For all the particles, the second (and subsequent) cycle shows virtually no hysteresis and tends to follow the first expansion.

Hysteresis effects in monolayers have been attributed to a number of causes: surface pressure gradient along the layer, irreversible loss of material, irreversible organization and motion of 

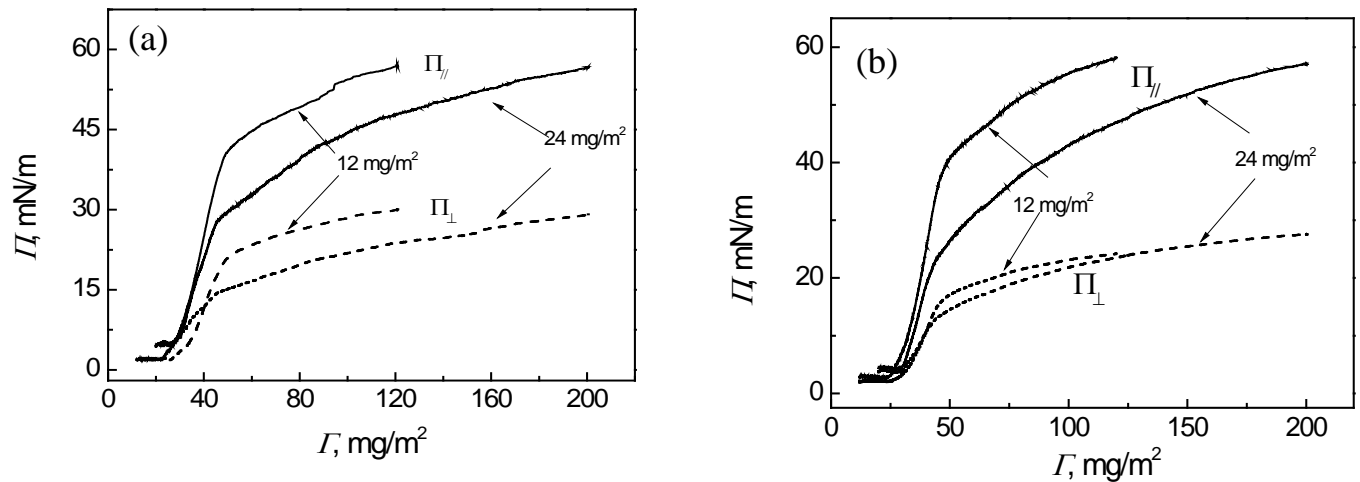

Figure 7. Effect of initial quantity of particles on layer properties. The barrier speed is $7 \mathrm{~cm}^{2} / \mathrm{min}$. (a) $\Pi-\Gamma$ isotherms for $20 \% \mathrm{SiOH}$ particle layer, (b) $\Pi-\Gamma$ isotherms for $34 \% \mathrm{SiOH}$ particle layer.

The $\Pi-\Gamma$ isotherms are strongly influenced by the initial surface particle concentration. The higher the initial $\Gamma_{0}$, the lower the surface pressure at large area, although the surface pressure at the end of compression is the same. The pressures at which buckling emerges decrease significantly with increasing $\Gamma_{0}$. These tendencies are consistent with the behavior of the deposited layers (uncompressed), which correspond to the largest particle quantity for a given concentration $\Gamma$. Similar initial particle quantity dependences were observed in other particle layers, possibly due to the existence of surface pressure gradients and non-homogeneous spreading which create defects in the layer [24]. The greater the amount of particles spread on the air-water interface, the larger the number of defects before compression. A recent paper also reported an effect of the amount spread on surface pressure, which was attributed to 
two-dimensional granular effects [29]. It is however unlikely that a similar interpretation could explain our observations, since the particles are not in close contact in the layers studied here, and granular friction is absent.

\subsubsection{Effect of trough length}

The surface pressure decreases when the length $L$ of the trough increases, keeping the linear velocity of the barrier $V$ constant (see Figure 8), as also reported in [29].

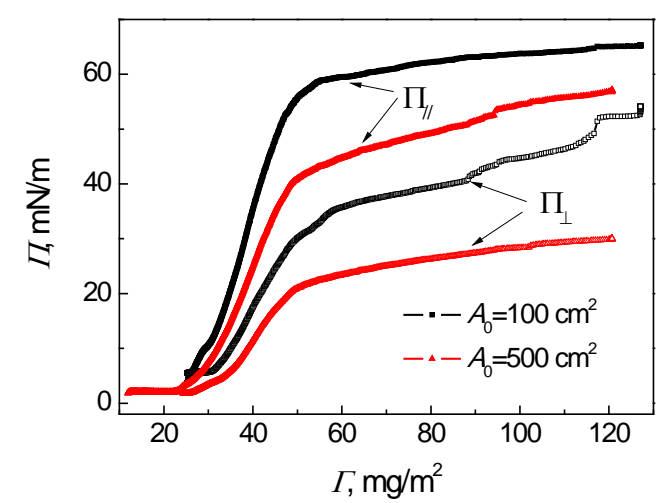

Figure 8. Effect of initial trough area on the $\Pi-\Gamma$ isotherms of silica particle layers possessing $20 \%$ $\mathrm{SiOH}$. The continuous barrier speed is $25 \mathrm{~cm}^{2} / \mathrm{min}$. Both troughs have the same width $(7 \mathrm{~cm})$.

This is equivalent to the dependence of surface pressure on the velocity of the barriers $d A / d t$ (Figure 5), since $d A / d t=L V$. Indeed, for constant $V$, the compression rate $(d A / d t) / A=V / L$, and $L$ is 5 times smaller in the small trough.

\subsection{Mechanical properties}

\subsubsection{Compression modulus - compressed and deposited layers}

We determined the shear elastic modulus $G$ of the layers using two orthogonal plates : $G=$ $\Gamma / 2 \partial\left(\Pi_{\| /}-\Pi_{\perp}\right) / \partial \Gamma$ as explained in a previous paper [16]. In this paper, we showed that the shear rate is not well defined in this method and since $G$ depends appreciably on shear rate, no reliable data can be obtained. The compression elastic modulus $E$ however should not be affected. This modulus can be obtained as: $E=\Gamma / 2 \partial\left(\Pi_{/ /}+\Pi_{\perp}\right) / \partial \Gamma[16]$. The experiments were performed for particles of different hydrophobicities and for both compressed and 
deposited layers. The moduli are maximum at the concentration $\Gamma$ just before buckling and we have plotted the corresponding values in Figure 9.

We observe an important dependence on the particle hydrophobicity, $E$ being the largest for $\mathrm{x}$ $\sim 34 \%$, the particles leading to the most stable foams. The results for compressed layers are qualitatively similar to those of ref. 18 although the values are somewhat different due to the fact that in this former work, only compressed layers were studied and one plate was used ( $E$ was calculated as $E=\Gamma \partial \Pi / \partial \Gamma)$.

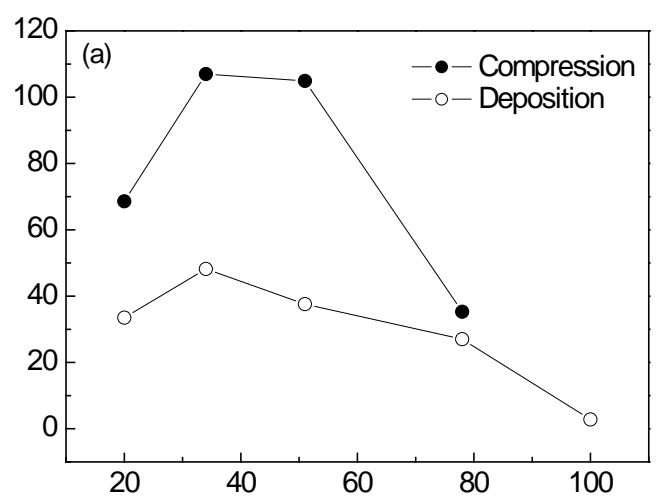

Figure 9. Maximum compression modulus as a function of particle hydrophobicity for compressed and deposited layers.

\subsubsection{Shear properties}

The shear properties of the deposited particle layers have also been studied using a shear rheometer. Note that it is not possible to install barriers and to study compressed layers in the rheometer used. We performed strain amplitude sweeps (with $\Gamma$ fixed at $50 \mathrm{mg} / \mathrm{m}^{2}$, which corresponds to the maximum surface pressure on most layers), during which the strain $\gamma_{0}$ was varied from 0.01 to $20 \%$ while the frequency was fixed at $2 \mathrm{~Hz}$. The $G^{\prime}$ and $G^{\prime \prime}$ define both parameters curves for different particle hydrophobicities are plotted in Figure 10.

The inset in Figure 10(a) shows a typical curve of $G^{\prime}$ and $G^{\prime \prime}$ versus $\gamma_{0}$. The strain $\gamma_{\mathrm{M}}$ at which $G^{\prime}=G^{\prime \prime}$ indicates the onset of fluidization of the surface layer, and is defined as the melting strain of the layer. 

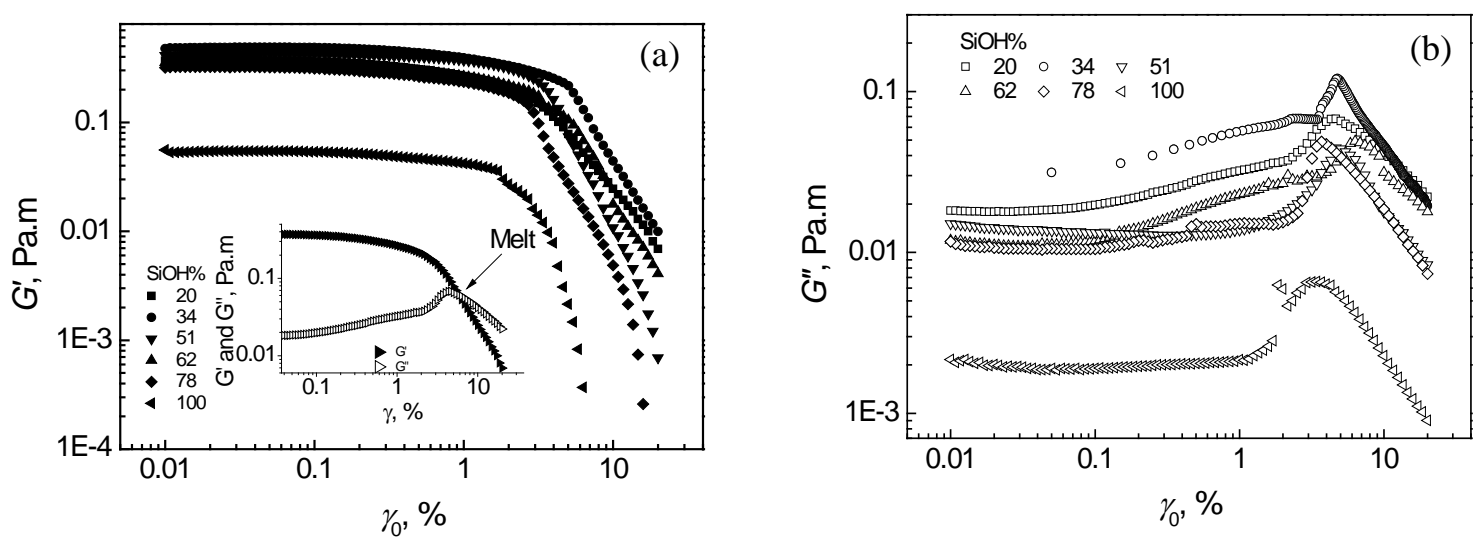

Figure 10. Variation of (a) $G^{\prime}$ and (b) $G^{\prime \prime}$ with strain $\gamma_{0}$ for different particle layers of varying hydrophobicity. The inset shows both $G^{\prime}$ and $G^{\prime \prime}$ versus $\gamma_{0}$ for $20 \% \mathrm{SiOH}$ particles. Frequency is $2 \mathrm{~Hz}$.

Figure 11 shows the interfacial shear stress $\sigma_{\mathrm{i}}$ during amplitude sweeps.

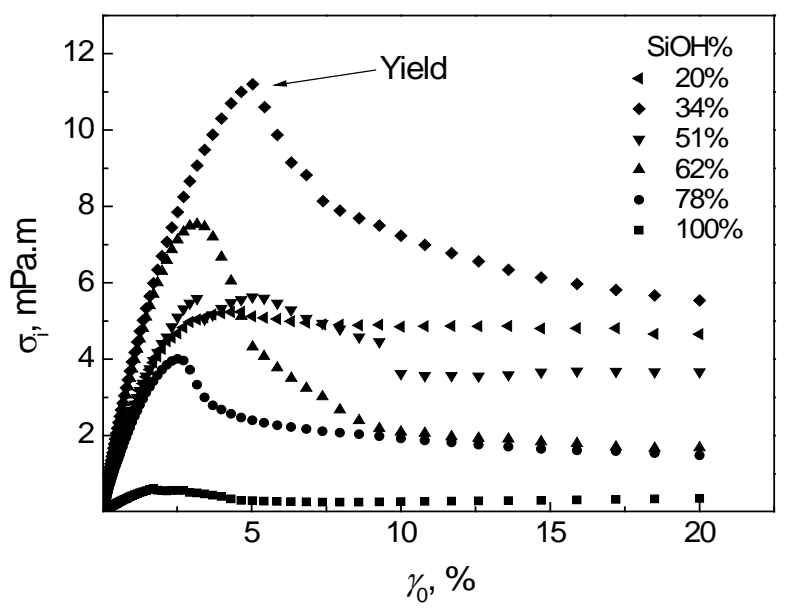

Figure 11. Interfacial stress $\sigma_{i}$ during amplitude sweep for different particle layers $\left(\Gamma=50 \mathrm{mg} / \mathrm{m}^{2}\right)$. In the small $\gamma_{0}$ region, $\sigma_{i}$ increases linearly, then decreases above a sufficiently large deformation which is called the yield stress $\gamma_{\mathrm{Y}}$. Frequency is $2 \mathrm{~Hz}$.

We mentioned in $\$ 3.1$ that the particle layer can self heal upon re-compression. The elastic shear modulus $G^{\prime}$ can similarly recover rapidly when the stress is released and the layers undergo a slow rejuvenation [19]. Figure 12 shows the time recovery of $G^{\prime}$ normalized by its initial value, i.e. $G_{\mathrm{R}}^{\prime}=G^{\prime} / G^{\prime}$. For $\Gamma=50 \mathrm{mg} / \mathrm{m}^{2}, G_{\mathrm{R}}{ }_{\mathrm{R}}(0)=0.89,0.76,0.70$ and 0.45 for $20 \%$, 34\%, 51\% and 62\% $\mathrm{SiOH}$ particle layers, respectively. One can see that the less dense layers heal faster as well as the more hydrophobic particle layers. 


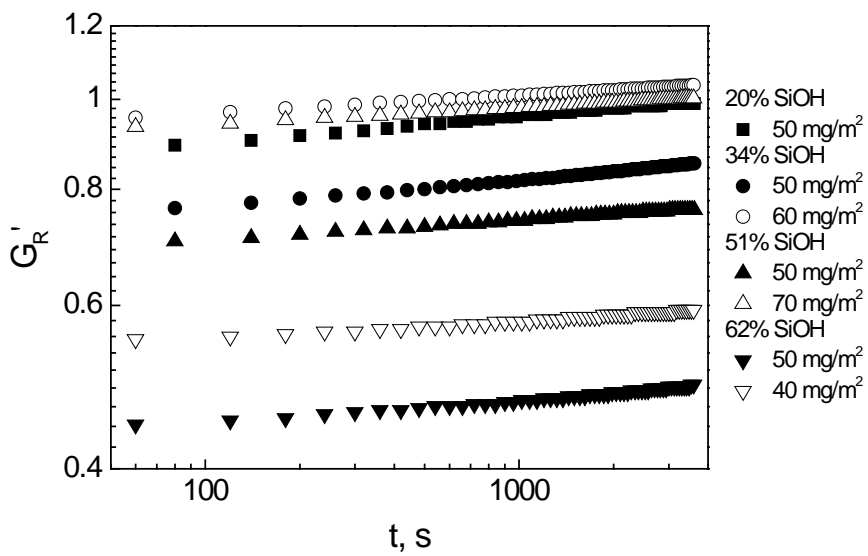

Figure 12. The healing dynamics of different particle layers. In order to avoid further fracturation of the layer, a small amplitude is used $\left(\gamma_{0}=0.01 \%\right)$. Frequency is $2 \mathrm{~Hz}$.

From Figure 4(b), $\Delta \Pi$ for layers of $34 \% \mathrm{SiOH}$ particles is the largest indicating it has the largest resistance to shear deformation. This is confirmed by the shear rheometer study, as shown in Figure 13 (a).
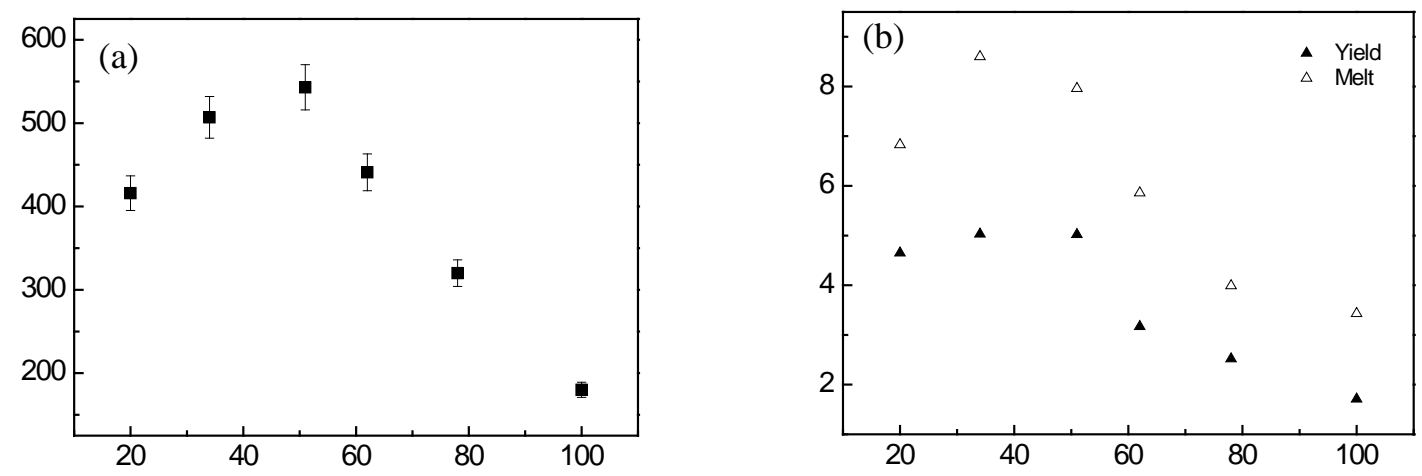

Figure 13. (a) $G^{\prime}$ versus $\mathrm{SiOH}$ content from an amplitude sweep with strain 1\% (in the linear region). (b) Yield and melting strain as a function of $\mathrm{SiOH}$ content on the particles.

The yield strain $\gamma_{Y}$ and melting strain $\gamma_{M}$ obtained by shear rheology are illustrated in Figure 13 (b). Both present a maximum at intermediate $\mathrm{SiOH}$ content. For particles of $34 \% \mathrm{SiOH}$, the yield strain $\gamma_{Y}$ is quite high at $5 \%$, much higher than that of $<1 \%$ for silver nanoparticles adsorbed at an oil-water interface [30]. However, it is close to that of monolayers of insoluble surfactants and proteins [31, 32]. Note that the shear moduli measured here are much larger than those of ref. 18 in which a different rheometer was used, which did not give access to 
large $G$ values and where measurements could only be performed at low $\Gamma$.

\section{Conclusions}

The work presented in this paper highlights the important role of the particle hydrophobicity, or equivalently the contact angle, in the properties of the monolayers that they form at the air-water interface. Although the behavior of the monolayers is qualitatively similar, important quantitative differences were measured.

The surface pressure measurements are consistent with the earlier ones on compressed layers of ref. 18. In particular, the phenomena of hysteresis, self healing and slow self healing have been observed for particles of all hydrophobicity as well. The surface domains are denser for particles of higher hydrophobicity. Buckling has also been observed but only for compressed layers; deposited layers rather form domains of larger thickness at high concentration. The surface pressure of the deposited layers is always smaller than that of compressed layers revealing that the degree of compaction of the particles is lower.

In order to better account for non-equilibrium effects, we have used two perpendicular Wilhelmy plates and observed a strong surface pressure anisotropy for all particles, which reflects the existence of finite shear moduli. Compression can generate a non-equilibrium configuration of the layer, which relaxes afterwards. The relaxation processes are extremely long, with times of the order of hours. They account for the puzzling roles of compression velocity, through trough length and amount of spread particles. Successively deposited layers appear to be closer to equilibrium.

The compression properties depend on the particle contact angle between air and water. The maximum compression elastic moduli $E_{\max }$ of both compressed layers and deposited layers is maximum around $\theta=90^{\circ}$. The results are consistent with the fact that the foamability and foam stability of aqueous foams made with the same particles possessing $34 \% \mathrm{SiOH}$ are the highest compared to those made with particles of other hydrophobicities. Indeed high elastic compression moduli slow down (eventually suppressing) coarsening [33] and coalescence [34]. 
The shear elastic moduli $G^{\prime}$ as well as the yield and melt strains also show maxima at $\theta \sim 90^{\circ}$. The shear properties might play a role in the foam properties too, but the exact mechanisms remain to be elucidated. Furthermore, compact particle layers exhibit non-linear behavior, such as fracturation, when large deformations are applied. The self healing properties of the layers may also contribute to foam stabilization.

\section{Acknowledgments}

D.Y. Zang thanks the Project of Sino-French Doctor's Institutes from CSC (China Scholarship Council) and Laboratoire de Physique des Solides for financial support. G. Delon is grateful to CNES and ESA for post-doctoral fellowships. CNES and ESA are also thanked for partial financial support of the experiments. We thank Wacker-Chemie (Burghausen) for the fumed silica particles. A. Maestro Martin and A.Stocco are acknowledged for many fruitful discussions.

\section{References}

[1] R.G. Alargova, D.S. Warhadpande, V.N. Paunov and O.D. Velev, Langmuir, 20, 10371 (2004).

[2] B.P. Binks and T.S. Horozov, Angew. Chem. Int. Ed., 44, 3722 (2005); T.S. Horozov, B.P. Binks, R. Aveyard and J.H. Clint, Colloids Surf. A, 282-283, 377 (2006).

[3] U.T. Gonzenbach, A.R. Studart, E. Tervoort and L.J. Gauckler, Angew. Chem. Int. Ed., 45, 3526 (2006).

[4] S. Fujii, A.J. Ryan and S.P. Armes, J. Am. Chem. Soc., 128, 7882 (2006).

[5] A. Cervantes Martinez, E. Rio, G. Delon, A. Saint-Jalmes, D. Langevin and B.P. Binks, Soft Matter, 4, 1531 (2008).

[6] W. Ramsden, Proc. Roy. Soc. A, 72, 156(1903).

[7] S.U. Pickering, J. Chem. Soc, 91, 2001(1907).

[8] T. Kostakis, R. Ettelaie and B. S. Murray, Langmuir, 22, 1273(2006).

[9] G. Gillies, M. Kappl and H.-J. Butt. Adv. Colloid Interface Sci. 114-115, 165 (2005).

[10]H. Hadjiiski, S. Tcholakova, I.B. Ivanov, T.D. Gurkov and E.F. Leonard, Langmuir 18, 127 (2002).

[11]V.N. Paunov, Langmuir, 19, 7970 (2003). 
[12]T. S. Horozov, D. A. Braz, P. D. I. Fletcher, B. P. Binks and J. H. Clint Langmuir, 24, 1678 (2008).

[13]D. Y. Zang, A. Stocco, D. Langevin, B. Wei and B.P. Binks, Phys. Chem. Chem. Phys., 11, 9522 (2009).

[14]A. Subramaniam, M. Abkarian, L. Mahadevan and H. Stone, Langmuir, 22, 10204 (2006).

[15]M. Abkarian, A. Subramaniam, S.-H. Kim, R. Larsen, S.-M. Yang and H. Stone, Phys. Rev. Lett., 99, 188301 (2007).

[16]D. Y. Zang, E. Rio, D. Langevin, B. Wei and B. P. Binks, Eur. Phys. J. E, 31,125 (2010).

[17]O. H. Soo-Gun and J. C. Slattery, J. Colloid Interface Sci., 67, 516 (1978).

[18]M. Safouane, D. Langevin and B.P. Binks, Langmuir, 23, 11546 (2007).

[19]D. Y. Zang, D. Langevin, B.P. Binks and B. Wei, Phys. Rev. E, 81, 011604 (2010).

[20]J.T. Petkov and T.D. Gurkov, Langmuir, 16, 3703 (2000).

[21]P. Cicuta and E.M. Terentjev, Eur. Phys. J. E, 16, 147 (2005).

[22]T.A.M. Ferenczi and P. Cicuta, J. Phys.: Condens. Matter, 17, S3445 (2005).

[23]P. G. de Gennes, Rev. Modern Phys. 71, S374 (1999)

[24]M. Maté, J. H. Fendler, J. J. Ramsden, J. Szalma and Z. Hórvölgyi, Langmuir, 14, 6501 (1998).

[25]P. Dynarowicz-Latka, A. Dhanabalan and O.N. Oliveira, J. Phys. Chem., 103, 5992 (1999).

[26]L. Gambut, J.-P. Chauvet, C. Garcia, B. Berge, A. Renault, S. Riviere, J. Meunier, and A. Collet, Langmuir, 12, 5407 (1996).

[27]A. Dhanabalan, N. Prasanth Kumar, S. Major and S. S. Talwar, Thin Solid Films, 327-329, 787 (1998).

[28]C. J. L. Constantino, A. Dhanabalan and O. N. Oliveira, Rev. Sci. Instr., 70, 3674 (1999).

[29]P. Cicuta and D. Vella, Phys. Rev. Lett., 102, 138302 (2009).

[30]R. Krishnaswamy, S. Majumdar, R. Ganapathy, V.V.Agarwal, A.K.Sood and C.N.R.Rao Langmuir, 23, 3084 (2007).

[31]P. Erni, P .Fischer and E .Windhab, Rev. Sci. Instrum., 74, 4916 (2003).

[32]P. Erni, P. Fischer, P.Heyer, E.J.Windhab, V.Kusnezov and J.Läuger Prog. Colloid Polym. Sci., 129, 16 (2004).

[33]M. B. J. Meinders and T. van Vliet, Adv. Colloid Interface Sci., 108, 119 (2004).

[34]P.G. de Gennes, Chem.Eng.Sci., 56, 5449 (2001). 
2) At the end of 2.2, please specify the value (or range) of compression speeds in the work.

Two barrier speeds: $7 \mathrm{~cm}^{2} / \mathrm{min}$ or $25 \mathrm{~cm}^{2} / \mathrm{min}$ were used. This was added in the text.

3) Beginning of 2.3, please clarify the position of the fixed barriers (fully open ?) in the successive deposition method. The geometry (aspect ratio) of the trough may be relevant.

Yes, the barriers were not fully opened. The effective area of the trough is $180 \mathrm{~cm} 2$. This was added in the text.

4) End of 2.3. Is the reported decrease of the pressure the effect normally observed because of evaporation of the subphase, or is the trough filling level controlled ? Only in the latter case I think it may be a real effect due to the particle layer.

We are not sure about the real reason behind the decrease of pressure. But possibly part of it is due to evaporation of the subphase since it cannot be completely prevented in the experiment.

5) Page 7. It could be useful to know the spread amount, or to remind the reader of the area fully open in caption of fig. 4 so this could be worked out.

The spread amount is $0.6 \mathrm{mg}$, the fully open area is $500 \mathrm{~cm}^{2}$. The latter was added in the caption

6) Section 3.2.1 the perpendicular and parallel pressure symbols should be defined better, either by reference to one of the previous papers, or by having a small diagram (which would also address point 1).

The captions were modified accordingly.

7) Figure 5, I would recommend moving the legend information currently on the plots to the caption, since this is the same information for all 4 panels. The caption information of $\mathrm{SiOH}$ percentage should go on the panels. This would help the reader in understanding more promptly the significance of the 4 panels. 
This was corrected

8) Section 3.2.4 reports the effect seen when spreading different amounts of material. The isotherms do not overlap when plotted as a function of surface concentration, and the measured bucking pressure falls when more material is spread. This is the same effect measured in ref [29], where in fact the trend of buckling pressure versus the bucking area showed a linear dependence. The authors may want to plot their data of bucking pressure obtained from Fig 7, in this way. In any case, reference should be made to [29] for this effect.

We do not have enough data to test the surface concentration dependence, but we know that the interpretation of ref 29 could not be valid in our case, since the surface coverage by particles at the buckling concentration is here only about $20 \%$.

9) Reference [29] is currently cited in 3.2.5. In this section, the influence of compression speed on the measured pressure is mentioned. However, in this paper at least, the authors have not tested the effect of changing compression speed with the same trough geometry. So I find this section a little confusing. Either: (a) the authors test that changing compression rate by a factor of 5 (independently of geometry) affects buckling pressure a lot, as implied in the current text; then this system is different from the one in ref [29]; or (b) the authors find that compression rate is not an important factor; then this sample is the same as ref [29], and the boundary condition effect described in [29] should be taken as a guide to describe the data of fig 7 and fig 8.

The troughs have the same width, this was added in the text; the data can be therefore compared without problem with the results of ref 29.

10) It would be useful to remind the reader in the figure captions that the experiments in Fig. 10 and Fig. 11 are done at $2 \mathrm{~Hz}$.

This was done.

11) Caption of Fig.12 should be expanded slightly (or in the main text) to describe better the experimental conditions during the "healing" measurement: strain amplitude, frequency, etc.

to avoid fracturation of the layer, the strain amplitude is very small(0.01\%), the frequency is $2 \mathrm{~Hz}$. This was added

12) In Figure 13, the guides to the eye should be removed.

This was done.

13) In acknowledgements, I believe the authors are thanking A. Maestro Martin.

Yes, in France we use only one name, but his second name was added. 